\title{
Controlling antibiotic usage - analysis of nationally published data from GP practices including demography, geography, comorbidity and prescribing factors highlights opportunities to reduce overall prescribing through changes in discretionary prescribing choices
}

\author{
Authors: Adrian Heald, ${ }^{\mathrm{A}}$ Mark Davies, ${ }^{\mathrm{B}}$ Mark Lunt, ${ }^{\mathrm{A}}$ Erin Fulton-McAlister, ${ }^{\mathrm{C}}$ Hussain Abid, ${ }^{\mathrm{D}}$ Tjeerd Van Staa, ${ }^{\mathrm{E}}$ \\ Simon Anderson ${ }^{F}$ and Michael Stedman ${ }^{B}$
}

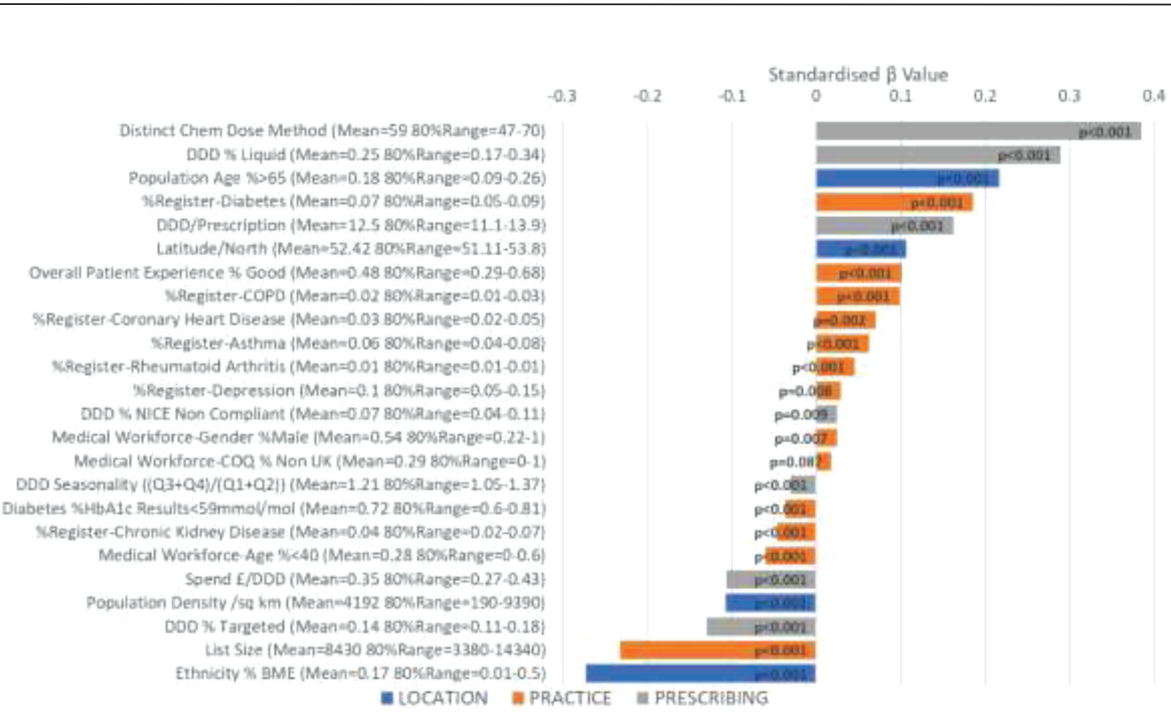

Fig 1. 2017-18 cross-sectional regression analysis (factors on the left side of the graph relate to less prescribing).
Antimicrobial resistance (AMR) is a critical and worsening public health threat driven by high volumes of use and inappropriate clinical targeting. Ecological studies show an association between antibiotic use and resistance rates. Moderation of antibiotic prescribing requires a deeper understanding of differences in local prescribing behaviours. The purpose of this study was to understand the factors in antibiotic prescribing determined by

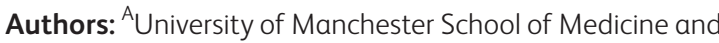
Manchester Academic Health Sciences Centre, Manchester,

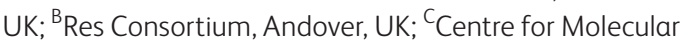
Bacteriology and Infection, London, UK; ${ }^{\mathrm{D}}$ Heart of England NHS Trust, Birmingham, UK; ${ }^{E}$ School of Health Sciences University of Manchester, Manchester, UK; FThe George Alleyne Chronic Disease Research Centre, Bridgetown, Barbados discretionary choice behaviour that may provide insights into enhanced methods to modify antibiotic usage and control AMR. Oral antibiotic prescribing information extracted from general practitioner (GP) prescribing data were aggregated by applying WHO Anatomical Therapeutic Chemical Classification (ATC) defined daily doses (DDD). Average annual antibiotic daily prescribing rate (AAADPR) was the sum of DDD of all oral antibiotics in each practice divided by practice population and 365 (days). AAADPR in each GP practice with $>2,000$ people in England in 2017-18 were linked by regression to other publicly available factors including demographics, geography, medical comorbidities, clinical performance, patient satisfaction, prescribing selection and medical workforce characteristics. Regression coefficients for those modifiable prescribing selection factors were applied to the difference between values for median and top decile practices to establish the overall reduction opportunities through changing prescribing behaviour. 
Fig 2. Trend in prescribing factors over last 5 years linked to model regression.

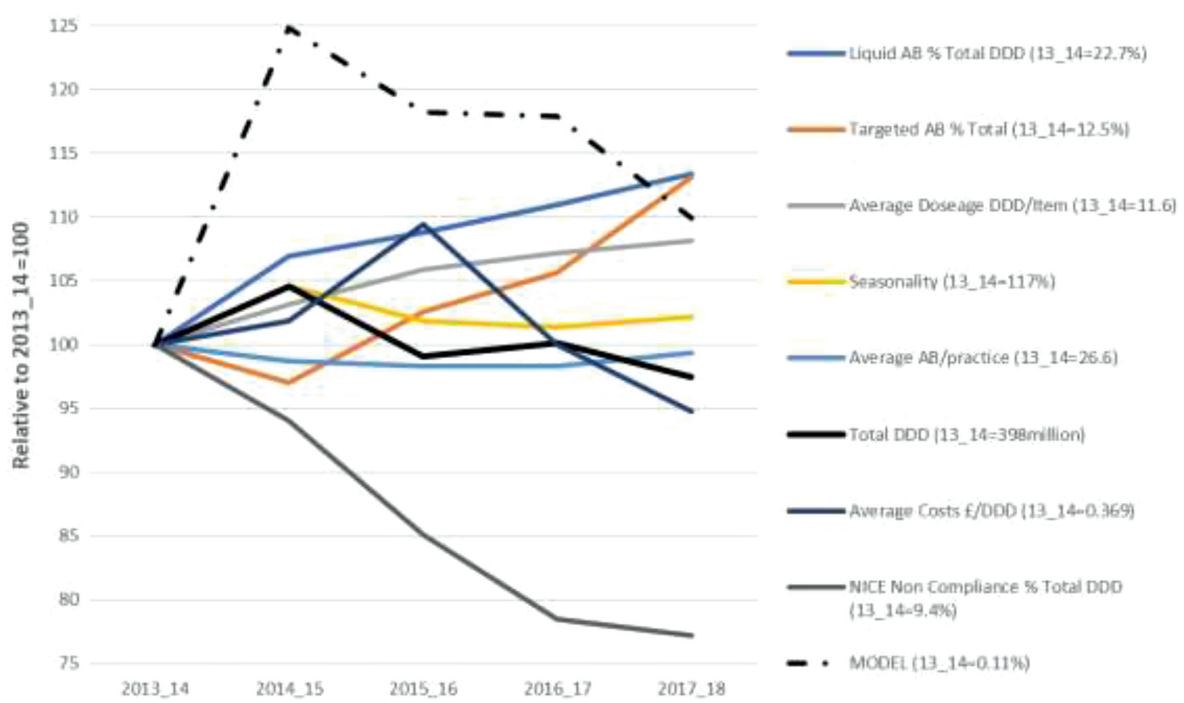

the overall prescribing. The model also suggests that if all practices achieved the level of prescribing modifiable factors of the top decile practices, the statistical model suggests that overall AAADPR would be reduced by up to a further $31 \%$.

We acknowledge that such analysis cannot infer causation and is ultimately associative in its nature. Demographics, location and medical condition of the population are major drivers in antibiotic prescribing in general practice. However prescribing selection plays a significant role and results from this analysis can provide benchmarks for both the non-modifiable and modifiable factors against which practices could evaluate their opportunities to reduce their antibiotic prescribing.

\section{Conflicts of interest}

None declared. 